Case Reports

\author{
Hari Poudel, MBBS, MS \\ Rajeev Bhandari, MBBS, MS \\ Bikesh Kumar Khambu, MBBS, MS, M.Ch \\ Rajendra Shrestha, MBBS, MS, PhD \\ Rajeev Jha, MBBS, MS, MCh \\ Prakash Bista, MBBS, MS, MCh \\ Address for correspondence: \\ Dr Hari Poudel, MBBS, MS. \\ National Academy of Medical Sciences \\ Bir Hospital, Kathmandu, Nepal \\ drharipoudel@yahoo.com
}

Date submitted : 18 June 2019

Date accepted : 31August 2019

\section{Schwannoma Presenting As Pituitary Macroadenoma: Case Report}

Schwannoma is common intracranial tumor. They are benign tumor arising from Cranial nerves or peripheral nerves. Majority of these tumors occur at Cerebellopontine angle. 54 year right handed lady presented with history of progressive loss of vision on both eyes for last five years. It started on her left eye initially pronounced on lateral field which gradually progressed to involve right eye. MRI of brain showed a well-defined mass of size $39 * 35 * 29$ $\mathrm{mm}$ in sellar region extending to suprasellar region with $\mathrm{T} 1$ isointensity and $\mathrm{T} 2$ hyperintensity. Endonasal trans-sphenoidal resection of lesion was done guided by navigation. Surprisingly histopathology turned out to be Schwanoma.

Key Words:Schwannoma, Pituitary, Sella, Tumor
$\mathrm{S}$ chwannoma is common intracranial tumor. They are benign tumor arising from Cranial nerves or peripheral nerves. Majority of these tumors occur at Cerebellopontine angle. ${ }^{1}$ There are rare case reports of Schwannoma elsewhere ${ }^{2-5,8,10}$. We present a case of pituitary fossaSchwannoma.

\section{Case Report}

54 year right handed lady presented with history of progressive loss of vision on both eyes for last five years. It started on her left eye initially pronounced on lateral field which gradually progressed to involve right eye. Over a duration of three years, she could barely notice moving objects in front of her eyes. However she could not see objects. She had on and off headache which was progressive in terms of intensity and duration. 10 days prior to presentation her vision further deteriorated and she had severe headache.

On examination, she was well oriented to time, place and person. Higher mental function was normal. Olfactory sense was absent in both nostril. Vision was only hand movement on both eyes. Fundoscopy revealed pallor more on temporal side bilaterally. Perimetry showed loss of temporal field on both eyes more on left. Rest of cranial nerve examination was normal. The results of motor and sensory examinations were unremarkable. There were no neurocutaneous markers evident.

Hormonal assay showed secondary hypothyroidism andhypocortisolism. Contrast enhanced MRI of brain 


\section{Poudel et al}
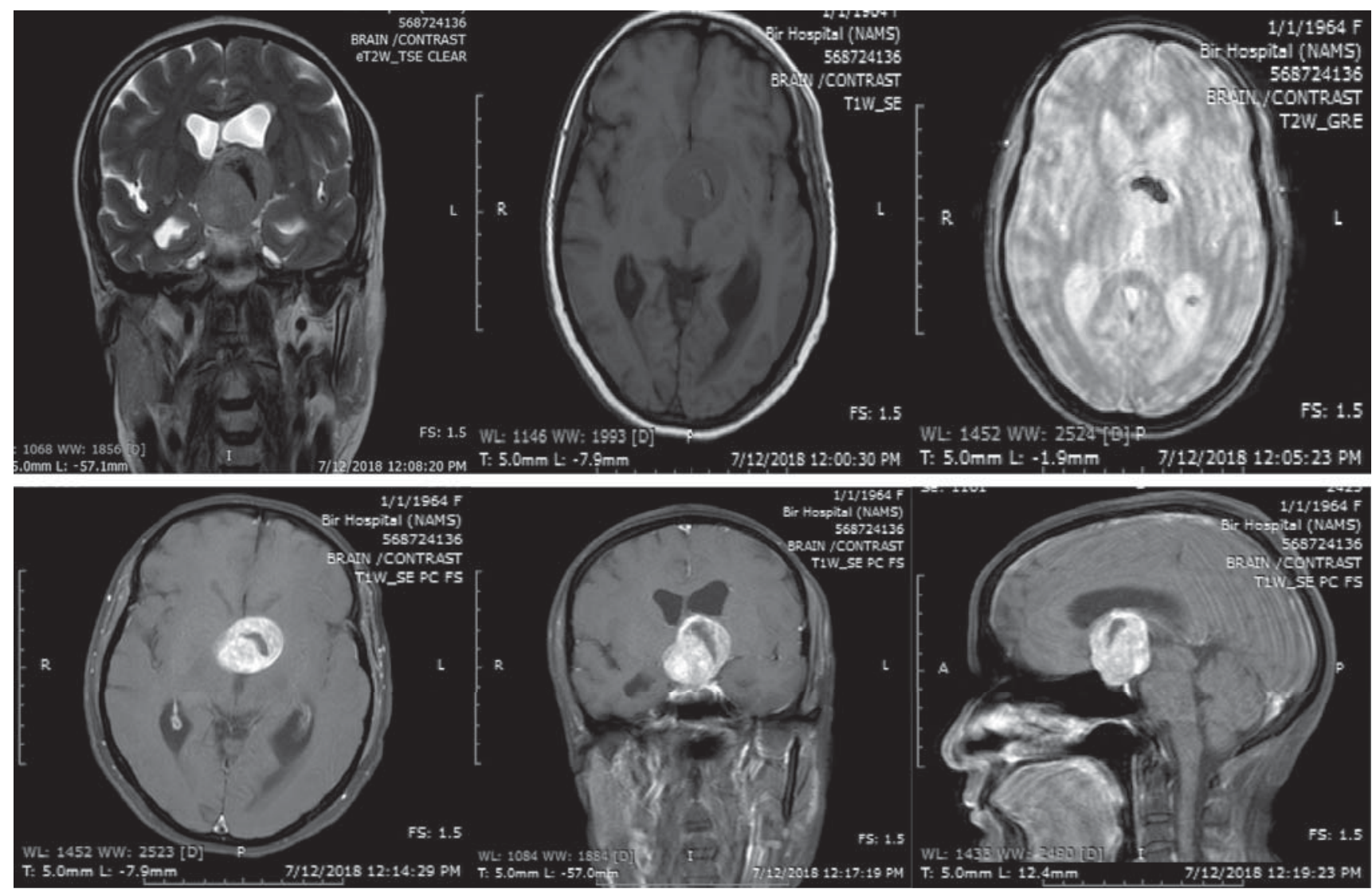

Figure 1:Coronal T2 weighted image showing well defined hyperintensesuprasellar\&sellar mass extending into B/L cavernous sinus displacing B/L ICA with distortion of left cavernous sinus, compression of optic chiasm \& compression of third ventricle with upstream hydrocephalus ventriculomegaly. There is low signal intensity focus within it, Middle image: axial T1 weighted image showing iso-intnese mass with low \& high signal focus.Right GRE image showing blooming artifacts (likely hemorrhage) in the area of T2WI low signal intensity and T1WI low \& high signal intensity.Post-contrast axial, coronal and sagittal image of same patient showing heterogeneous enhancement of supra-sellar and sellar mass with non-enhancing focus.

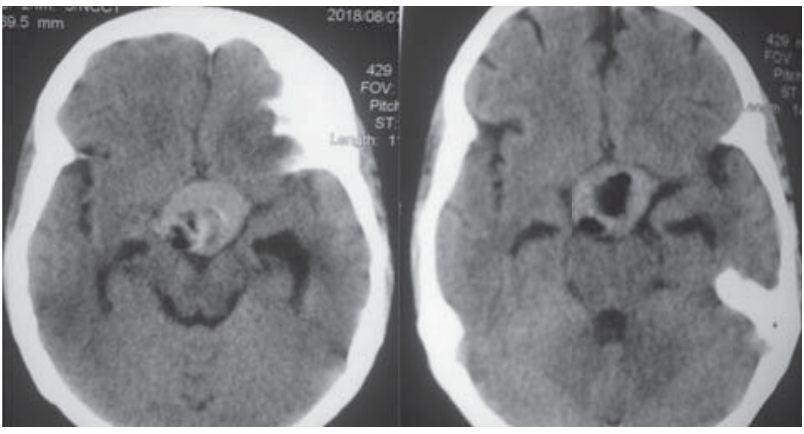

Figure 2: Pre and post-operative CT showing significant reduction of mass after surgery

with thin cuts on sellar region was done. There was well defined mass of size $39 * 35 * 29 \mathrm{~mm}$ in sellar region extending to suprasellar region with $\mathrm{T} 1$ isointensity and $\mathrm{T} 2$ hyperintensity. Lesion had heterogeneous enhancement on contrast injection. Lesion was extending to anterior aspect of third ventricle leading to hydrocephalus; laterally into bilateral cavernous sinus with lateral displacement of cavernous portion of ICA. These features were consistent with pituitary macroadenoma

We proceeded with Endonasal trans-sphenoidal resection of lesion. Intraoperatively the lesion was soft to firm, suckable, with moderate vascularity. Safe maximal resection was carried out guided by navigation. Tumor at cavernous sinus and lateral recess was left for safety. Surprisingly histopathology turned out to be Schwanoma. We further proceeded to resect remaining tumortransnasally. Histopathology of the specimen was studied. Patient developed Meningitis on postoperative period which responded well to intravenous antibiotics. Vision did not improve following surgery. Patient was discharged home. 


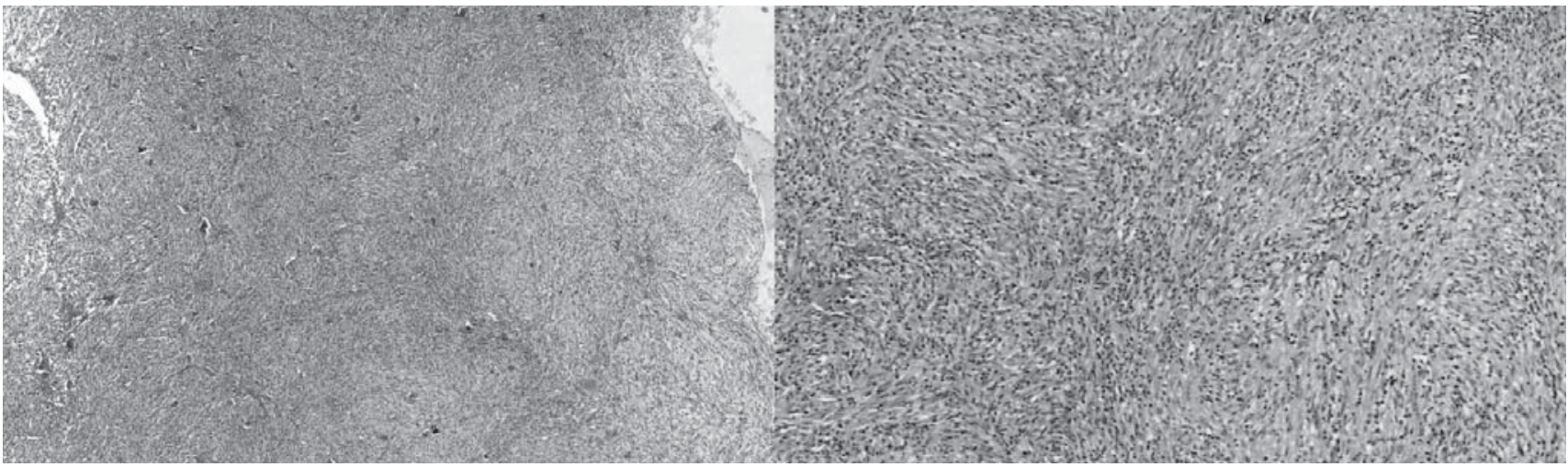

Figure 3: Cellular Antoni A areas showing spindle cells arranged in fascicles having elongated nuclei arranged in small interlacing bundles

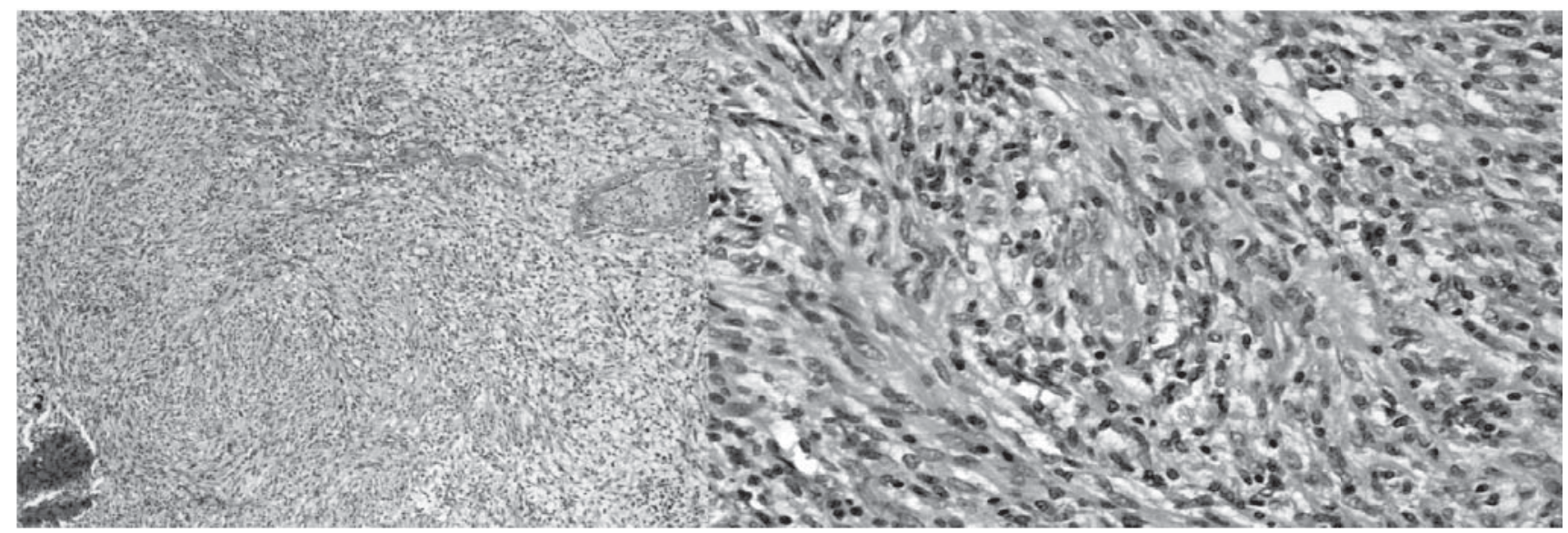

Figure 4: Hypocellular Antoni B where there is less cellularity with variable lipidization, areas showing both compact and less cellular areas(left). High power view of compact hyper cellular area, where cells have round to spindle shaped nuclei with tapered ends and having moderate amount of cytoplasm(right).

\section{Discussion}

Schwannoma accounted for about 6.8-8.0 per cent of all primaryintracranialtumours of which 80-90 percent were vestibular schwannomas. ${ }^{1}$ Other cranial nerve involvement are rare and limited to case reports. Gibson reported a case of Temporal lobe Schwannoma for the first time as intracerebral mass. ${ }^{9}$ Schwannoma may rarely presents as meningioma on preoperative imagings. IntrasellarSchwanoma is a rare presentation. It has been reported in about 30 cases have been reported till now in literature. ${ }^{6,7}$ Pituitary adenoma, craniopharyngioma, meningioma amongst others are common sellar pathology. Clinical presentation is similar to pituitary adenoma.

Schwannomas generally arise in sensory, rather than motor; cranial or spinal nerves. Intraparenchymal schwannomas arise very rarely. They usually present with sensory symptoms. However index case had a presentation of mass effect in form of vision loss and compression to pituitary probably owing to narrow sellar confinements. This led to our preoperative suspicion of pituitary adenoma. Retrospectively, the intralesional T1 hypo to iso intensity $\mathrm{T} 2$ hyperintensities represented cystic degeneration.

Schwannoma are typically well-encapsulated globoid lesions with a soft, rubbery texture. Unlike neurofibromas, they tend to leave the parent nerve as splayed fibres or an intact bundle around the tumour. As we approached the tumor presuming it to be a pituitary adenoma, Gross tumor characterstics could not be made out. As with other mass lesions, timely intervention is key to reversal of deficit. Vision could not be restored as there was optic atrophy. However hormonal remission was achieved with surgery

\section{Conclusion}

Schwannoma can rarely present as sellar mass. Safe maximal resection remains the optimaltreatment. 


\section{References:}

1. D’Alessandro G, Di Giovanni M, Iannizzi L, Guidetti E, Bottacchi E. Epidemiology of primary intracranial tumors in the valle d'aosta during the 6-year period 1986-1991. Neuroepidemiology 14(3): 139-46. (Italy), 1995

2. Khursheed N, Rumana M, Ramzan A, Furqan N, Abrar W, Salma B. Frontal intraparenchymal schwannoma. Journal of Clinical Neuroscience 18 (3):411-413, 2011

3. Lee S, Park SH, Chung CK. Supratentorial intracerebral schwannoma: Its fate and proper management. J Korean Neurosurg Soc 54 (4):340-3, 2013

4. Luo W, Ren X, Chen S, Liu H, Sui D, Lin S. Intracranial intraparenchymal and intraventricular schwannomas: Report of 18 cases. Clin Neurol Neurosurg 115 (7):1052-7,2013

5. Ma L, Yang SX, Wang YR. Intracerebral schwannoma mimicking parasagittal meningioma. Journal of Craniofacial Surgery 24 (6):e541-e543,2013
6. Mohammed S, Kovacs K, Munoz D, Cusimano MD.A short illustrated review of sellar region schwannomas. Acta Neurochir (Wien) 152 (5):885-91,2010.

7. Oishi T, Takehara S, Yamamura Y, Tomida M, Ito S, Kuriki K, et al. "Pure" Suprasellar Schwannoma Presented with Communicating Hydrocephalus: A Case Report. NMC case Rep J;4 (3):83-7,2017

8. Srinivas R, Krupashankar D, Shasi V. Case Report Intracerebral Schwannoma in a 16-Year-Old Girl. A Case Report and Review of the Literature. Case Rep Neurol Med:171494,2013

9. Takei H, Schmiege L, Buckleair L, Goodman JC, Powell SZ. Intracerebral schwannoma clinically and radiologically mimicking meningioma. Pathol Int 55 (8):514-9,2005

10. Zhang J, Xu S, Lui X, et al. Intrasellar and Suprasellar Schwannoma misdiagnosed as pituitary macroadenoma. World Neurosurg 28 (1): 127-135, 2016 